\title{
Article
}

\section{Nail Melatonin Content: A Suitable Non-Invasive Marker of Melatonin Production}

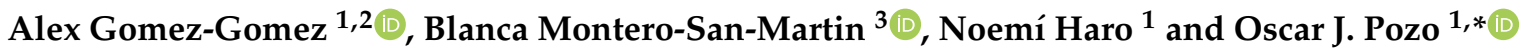 \\ 1 Integrative Pharmacology and Systems Neuroscience Group, Institut de l'Hospital del Mar d'Investigacions \\ Mèdiques (IMIM), Doctor Aiguader 88, 08003 Barcelona, Spain; agomez@imim.es (A.G.-G.); \\ nharo@imim.es (N.H.) \\ 2 Department of Experimental and Health Sciences, University Pompeu Fabra (CEXS-UPF), \\ Doctor Aiguader 88, 08003 Barcelona, Spain \\ 3 Laboratory Medicine, La Paz University Hospital, Paseo de la Castellana 261, 28046 Madrid, Spain; \\ blancamontero1912@gmail.com \\ * Correspondence: opozo@imim.es; Tel.: +34-93-316-04-80
}

\section{check for}

updates

Citation: Gomez-Gomez, A.; Montero-San-Martin, B.; Haro, N.; Pozo, O.J. Nail Melatonin Content: A Suitable Non-Invasive Marker of Melatonin Production. Int. J. Mol. Sci. 2021, 22, 921. https://doi.org/ $10.3390 /$ ijms22020921

Received: 22 December 2020

Accepted: 12 January 2021

Published: 18 January 2021

Publisher's Note: MDPI stays neutral with regard to jurisdictional claims in published maps and institutional affiliations.

Copyright: (c) 2021 by the authors. Licensee MDPI, Basel, Switzerland. This article is an open access article distributed under the terms and conditions of the Creative Commons Attribution (CC BY) license (https:// creativecommons.org/licenses/by/ $4.0 /)$.

\begin{abstract}
Melatonin plays multiple physiological roles in the human body. Evaluation of melatonin production by the determination of urinary 6-sulfatoxymelatonin in 24-h samples has important drawbacks which hinder the successful evaluation of melatonin production in large cohorts. Here, we evaluated the potential of nail analysis for estimating melatonin production. Firstly, mass spectrometry methodology for the determination of melatonin in nails was optimized and successfully validated. The method was found to be linear in the range $6.5-830 \mathrm{fg} / \mathrm{mg}$ with intraday and interday accuracy in the range 100-104\%, precision below $15 \%$ and a LOD of $3.5 \mathrm{fg} / \mathrm{mg}$. Secondly, nail melatonin concentrations from 84 volunteers (age 5-96) were determined. The expected correlation between melatonin and age was obtained (correlation coefficient $-0.615 ; p<0.001$ ). Additionally, we showed that fingernails are preferable to toenails to determine nail melatonin content. Finally, fingernails collected for 180 days after melatonin administration (two volunteers, $1.9 \mathrm{mg} / \mathrm{night}$ during 5 days) were analyzed. Nail melatonin concentrations immediately rose after administration and went back to pre-administration values after $\approx 100$ days in both volunteers. Our results suggest that melatonin determination in nails is a suitable non-invasive tool for the estimation of global melatonin production. Due to the easy collection and storage of nails, the long-term information obtained and the multiple functions of melatonin, nail melatonin content might complement dim light melatonin onset, which is commonly measured from plasma/saliva samples, paving the way for melatonin research.
\end{abstract}

Keywords: melatonin; nails; fingernails; aging; liquid chromatography; mass spectrometry

\section{Introduction}

Melatonin is a hormone mainly released by the pineal gland at nighttime [1]. The main role of melatonin is the regulation of the sleep-wake cycle, although it plays additional roles, such as being an antioxidant by stimulating the synthesis of glutathione [2], and by scavenging hydroxyl and peroxyl radicals and stimulating superoxide dismutase, glutathione peroxidase and catalase [3,4]. Other functions of melatonin are related to body weight regulation [5], immune system maintenance [6] and reproduction [7,8].

Melatonin underproduction has been related to different types of dementia $[9,10]$, severe pain [11], cancer [12,13], diabetes type 2 [14] and other diseases [15-18]. Melatonin synthesis is also highly related to age. The levels of melatonin are decreased gradually over the life-span and are directly related to lowered sleep efficacy $[19,20]$. In fact, some of melatonin's actions have been reported to be beneficial for the process of aging [21-23].

Due to the important roles of melatonin, suitable strategies able to evaluate melatonin production are required. Melatonin determination presents important analytical 
challenges. Thus, researchers must measure levels at the femtomole range in the presence of compounds of similar molecular structure in complex matrices. Poor methodologies can imply the publication of studies that are seriously flawed [24,25].

The proper interpretation of the results is as important as the reliable analytical determination. Thus, single-point determinations of melatonin in either saliva or plasma are meaningless, and their use should be avoided [26]. Melatonin determination in serial plasma/saliva samples is preferred. This approach is commonly used to obtain the dim light melatonin onset (DLMO), the most accurate marker for assessing the circadian pacemaker [1]. The estimation of the global production of melatonin is commonly performed by the determination of urinary 6-sulfatoxymelatonin in 24-h samples [27]. Although this approach has been shown to be useful in controlled scenarios $[28,29]$, its practical application to studies based on large cohorts of volunteers is limited by the tediousness of sample self-collection. Additionally, the 24-h concentration of urinary 6-sulfatoxymelatonin only provides information about the melatonin production in the last day.

Therefore, new alternatives are required in order to establish a global production of melatonin, especially in large-cohort studies. A potential option might be the determination of melatonin in other matrices such as nails. Nails provide a unique feature. At a grow rate around $3 \mathrm{~mm} /$ month [30], fingernail samples can provide long-term information with the simplest sampling and storage. Nails have already been used for the evaluation of chronic stress by successfully correlating nail cortisol and the perceived stress scale [31]. Additionally, the usefulness of nails to determine the administration of drugs of abuse, e.g., benzodiazepines, tetrahydrocannabinol or cocaine, has also been demonstrated [32] Therefore, the potential determination of melatonin in nails may provide information about the global melatonin production decreasing the sampling complexity and increasing the period under evaluation.

The main purpose of our research was to explore the potential of the determination of melatonin in nails as an alternative for the evaluation of global melatonin production. Firstly, we optimized and validated a liquid chromatography-tandem mass spectrometry (LC-MS/MS) method able to determine the low melatonin concentrations in nails. We determined the nails melatonin concentrations in samples collected from 84 healthy volunteers. We also explored the potential usefulness of toenails by analyzing both fingernails and toenails collected from 22 healthy volunteers. Finally, we established the biological meaning of the determination by evaluating the period in which melatonin administration was detectable in nails.

\section{Results}

\subsection{Method Development}

When using an Acquity BEH C18 column $(100 \mathrm{~mm} \times 2.1 \mathrm{~mm}$ i.d., $1.7 \mu \mathrm{m})($ Waters Associates), the presence of a ubiquitous interference hindered the proper quantification of melatonin by acquiring the most abundant transition $(233>174)$. The acquisition of the secondary transition $(233>159)$ helped to establish the presence of that interference by comparing the ion ratio of the sample with the one of a solvent standard (Figure 1).

A more adequate separation was obtained when using an Acquity CSH C18 column (Figure 1) favoring the quantification of melatonin by the acquisition of the most abundant transition $(233>174)$.

Obtaining the ion ratios between the two selected transitions (273 > 174 and $273>159)$ was required to discern the presence of endogenous interferences. The low abundance of the qualitative transition $(273>159)$ hampered getting this ratio in low concentrated samples. For that reason, we sum up 10 qualitative transitions, increasing the sensitivity and allowing one to properly establish the ion ratios (Figure S1).

\subsection{Method Validation}

\subsubsection{Validation Using Melatonin-Free Nails}

Validation results using melatonin-free nails are depicted in Table 1. The method was found to be linear in the range $6.5-830 \mathrm{fg} / \mathrm{mg}$. The method was found to be accurate 
$(103-115 \%)$ and precise (coefficient of variation $<8 \%(C V<8 \%))$ at four different levels. A limit of detection (LOD) of $3.5 \mathrm{fg} / \mathrm{mg}$ was established for the method.

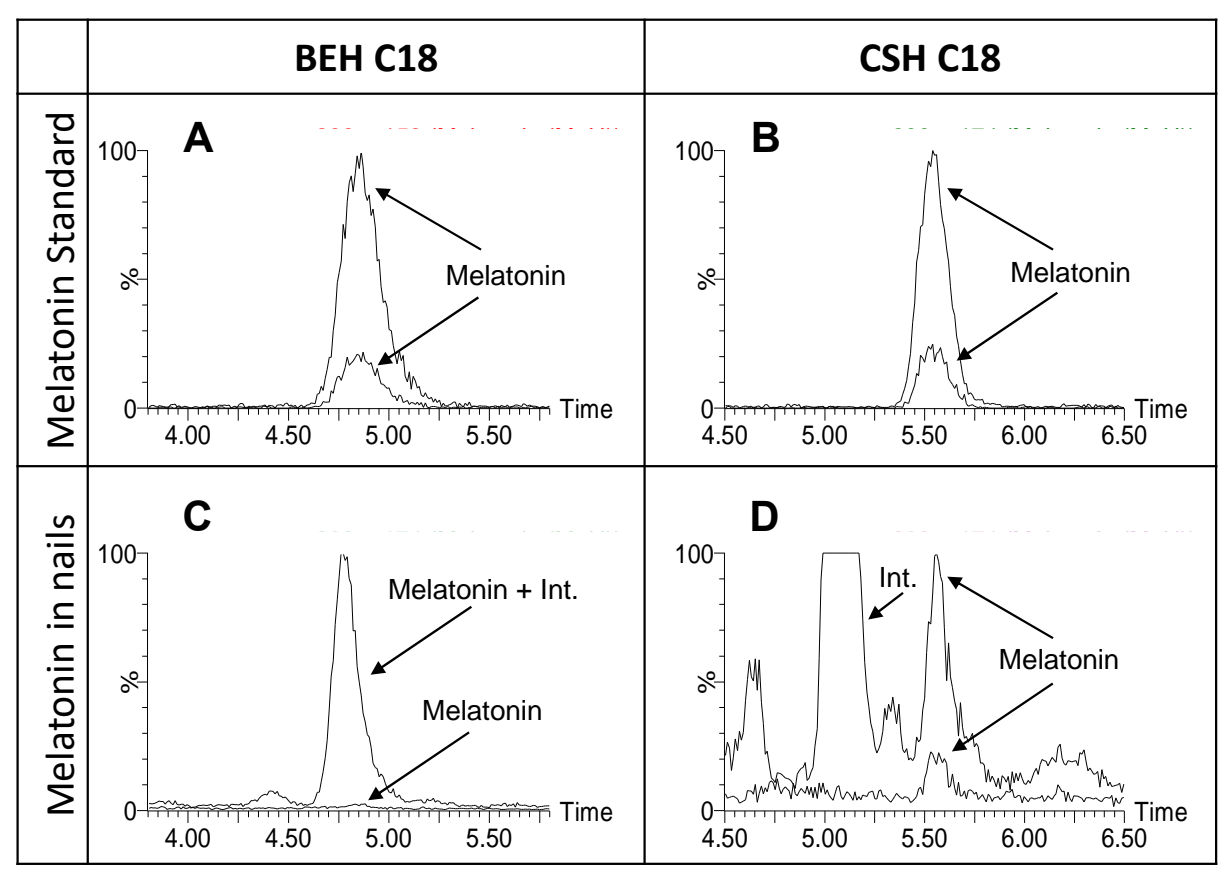

Figure 1. Relevance of chromatography in the LC-MS/MS detection of nail melatonin content. LCMS/MS chromatograms for the two selected transitions of melatonin (233 > 174 and $233>159)$ in standard (250 $\left.\mathrm{pg} \mathrm{mL}^{-1}\right)$ (A) and (B) and in a nail sample (31 fg/mg) (C,D). Chromatograms obtained by using two different chromatographic columns, BEH C18 (A,C) and CSH Phenyl-Hexyl (B,D). The time in the $\mathrm{x}$ axis is expressed in minutes. Abbreviation: Int.: interferent.

Table 1. Method validation parameters in melatonin-free matrix and real nail matrix. Calculated melatonin-free matrix parameters were linearity (in terms of weighting and range), LLOQ, LQC, MQC, HQC and LOD (in terms of accuracy and $\mathrm{CV}$ ). Calculated real nail matrix parameters were within-run and total accuracy and CV; matrix effect (in terms of recovery and $\mathrm{CV}$ ); the endogenous range of selected samples' melatonin; and specificity of the method by comparing standard and samples. Units of range, level, LOD and concentration are $\mathrm{fg} / \mathrm{mg}$.

\begin{tabular}{|c|c|c|c|c|c|c|}
\hline \multicolumn{7}{|c|}{ Melatonin-Free Matrix (CDER Regulations) } \\
\hline \multirow{3}{*}{ Linearity } & Weight. & Range (fg/mg) & & & & \\
\hline & $1 / x$ & $6.5-830$ & & & & \\
\hline & LLOQ & LQC & MQC & HQC & LOD & \\
\hline Level & 6.5 & 13 & 0.104 & 830 & 3.5 & \\
\hline Acc. $(\%)$ & 113 & 115 & 104 & 103 & - & \\
\hline CV $(\%)$ & 8 & 6 & 4 & 4 & - & \\
\hline \multicolumn{7}{|c|}{ Real nail matrix (Standard additions) } \\
\hline & Within-run $(n=6)$ & Total $(n=12)$ & & M.E. $(n=6)$ & & Endog Range \\
\hline Acc. $(\%)$ & 100 & 104 & Rec. $(\%)$ & 105 & Conc. & $30-110$ \\
\hline $\mathrm{CV}(\%)$ & 10 & 13 & $\mathrm{CV}(\%)$ & 7 & $(\mathrm{fg} / \mathrm{mg})$ & \\
\hline \multicolumn{7}{|c|}{ Specificity } \\
\hline & Standard & Sample 1 & Sample 2 & Sample 3 & Sample 4 & Sample 5 \\
\hline $\mathrm{Q} / \mathrm{q}$ ratio & 0.22 & 0.23 & 0.21 & 0.24 & 0.23 & 0.22 \\
\hline
\end{tabular}

Abbreviations: CDER: Center for Drug Evaluation and Research; Weight: weighting; LLOQ: lowest limit of quantification; LQC: lowest QC; MQC: medium QC; HQC: highest QC; LOD: limit of detection; Acc.: accuracy; CV: coefficient of variation; M.E.: matrix effect; Endog Range: endogenous range; Rec.: recovery; Conc: concentration; Q/q: ion ratio between quantitative transition (233 > 174) and qualitative transition $(233>159)$. 


\subsubsection{Validation Using Real Nail Matrix}

Validation results by standard additions using real nail matrix are summarized in Table 1. Melatonin levels of the samples used for standard additions were in the concentration range $30-110 \mathrm{fg} / \mathrm{mg}$. Both intraday (accuracy 100\%, CV =10\%) and interday (accuracy $104 \%, C V=13 \%$ ) results confirm the suitability of the method to determine nail melatonin concentrations. The method was found to be specific (difference in ion ratios $<10 \%$ in all samples tested) with no remarkable matrix effects (recovery 105\%, CV $=7 \%$ ).

\subsection{Method Application}

2.3.1. Establishment of Endogenous Levels of Fingernail Melatonin

The developed method was able to determine the endogenous concentrations in 84 fingernails samples (Table S1). Melatonin concentrations ranged from $58 \mathrm{fg} / \mathrm{mg}$ to $208 \mathrm{fg} / \mathrm{mg}$ for volunteers 5-20 years old (average $123 \mathrm{fg} / \mathrm{mg}$ for females and $130 \mathrm{fg} / \mathrm{mg}$ for males), from $26 \mathrm{fg} / \mathrm{mg}$ to $250 \mathrm{fg} / \mathrm{mg}$ for volunteers $31-40$ years old (average $88 \mathrm{fg} / \mathrm{mg}$ for females and $91 \mathrm{fg} / \mathrm{mg}$ for males), from $18 \mathrm{fg} / \mathrm{mg}$ to $117 \mathrm{fg} / \mathrm{mg}$ for volunteers $41-60$ years old (average $50 \mathrm{fg} / \mathrm{mg}$ for females and $48 \mathrm{fg} / \mathrm{mg}$ for males) and from $12 \mathrm{fg} / \mathrm{mg}$ to $89 \mathrm{fg} / \mathrm{mg}$ for volunteers $>60$ years old (average $50 \mathrm{fg} / \mathrm{mg}$ for females and $34 \mathrm{fg} / \mathrm{mg}$ for males) (Figure 2A,B and Table S1). No significant differences were obtained by gender (Figure 2A), but significant differences were observed by age (Figure 2B). We also found a statistically significant decrease (Pearson coefficient $-0.615, p<0.001$ ) of nail melatonin levels with age (Figure $2 \mathrm{C}$ ) with a clear decrease until c.a. forty years of age but after concentrations seem to remain constant (Figure 2).

\subsubsection{Potential of Toenails for Melatonin Determination}

We analyzed the melatonin content in both the fingernails and the toenails collected from 22 healthy volunteers. Our results showed that comparable levels (between $80 \%$ and $120 \%$ of the fingernail result) were obtained only for six volunteers (Figure 3, Table S2). Four volunteers showed higher concentrations (150-375\%) in toenails than in fingernails. Most of the volunteers (12 out of 22) exhibited lower levels (30-70\%) in toenails than in fingernails. Remarkably, the correlation between melatonin production and age observed when analyzing fingernails disappeared when using data from toenails.

\subsubsection{Effect of Exogenous Melatonin Administration in the Fingernail's Melatonin Levels}

Melatonin was evaluated in two volunteers' fingernails during a period of 180 days after melatonin intake. The first day after melatonin administration was considered as day 1. Basal melatonin fingernail levels of volunteers were determined in three samples collected pre-administration (samples represented between day -10 and day 0 ) and constant values were obtained for both volunteers (Figure 4). Melatonin administration was detected in the sample collected the day after the administration with a clear increase in the nail melatonin levels (Figure 4). The melatonin levels increased and reached their maximum peak during the administration period (day 5 for the male volunteer and day 2 for the female volunteer). After reaching their maximum, nail melatonin levels gradually decreased until returning back to basal levels after 109 days. 


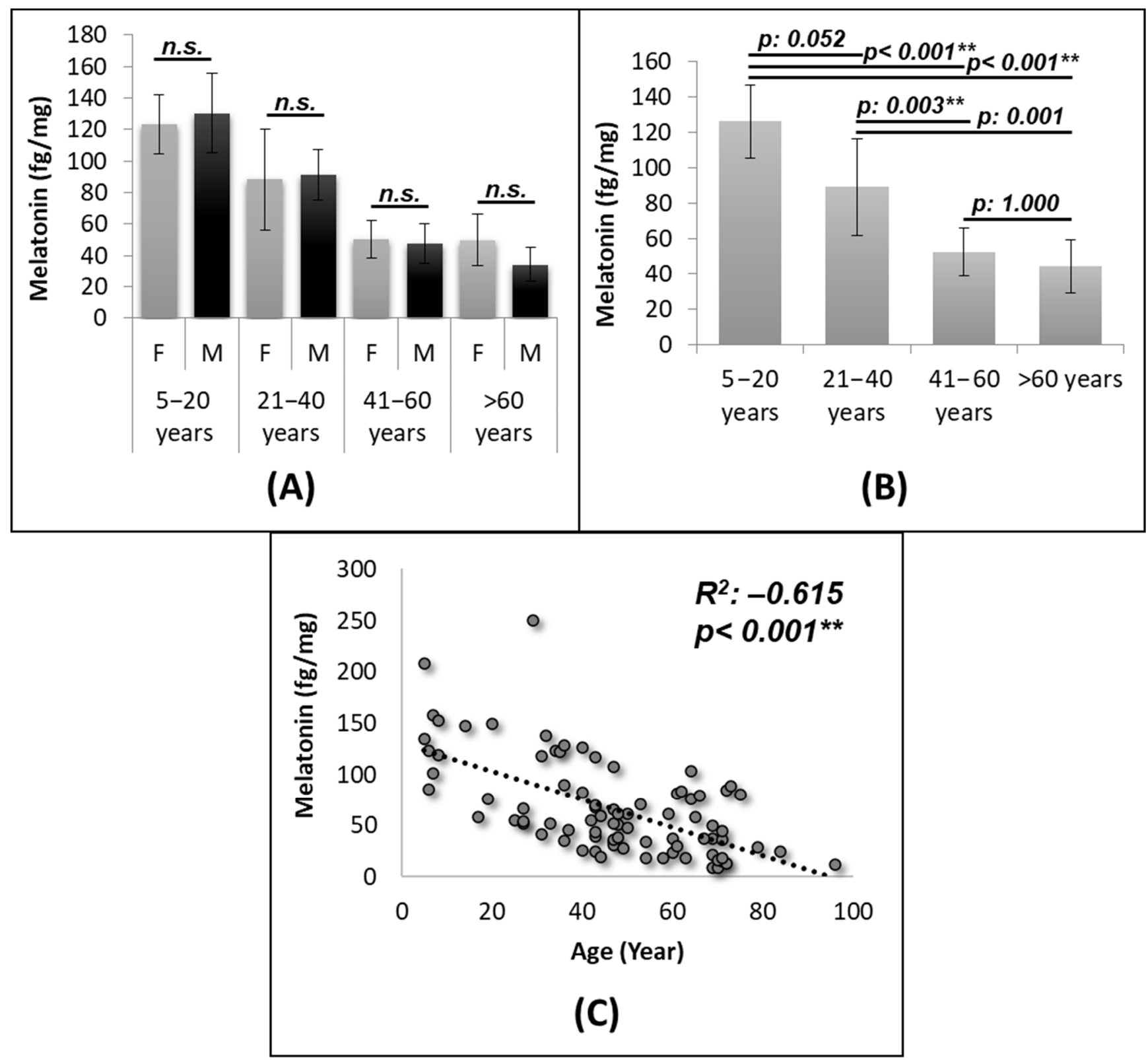

Figure 2. Fingernail melatonin concentrations are able to determine the gradual decrease of melatonin production over the life-span. (A) Gender stratified differences in the established age ranges: range 5-20, females $(n=6)$ and males $(n=5)$; range $21-40$, females $(n=11)$ and males $(n=5)$; range $41-60$, females $(n=14)$ and males $(n=15)$; range $>60$, females $(n=19)$ and males $(n=9)$. (B) Differences in age ranges when considering both genders together: range 5-20 $(n=11)$; range 21-40 $(n=16)$; range 41-60 $(n=29)$; range $>60(n=28)$. (C) Correlation between fingernail melatonin concentrations and age. ** Statistically significant differences $(p<0.01)$. 


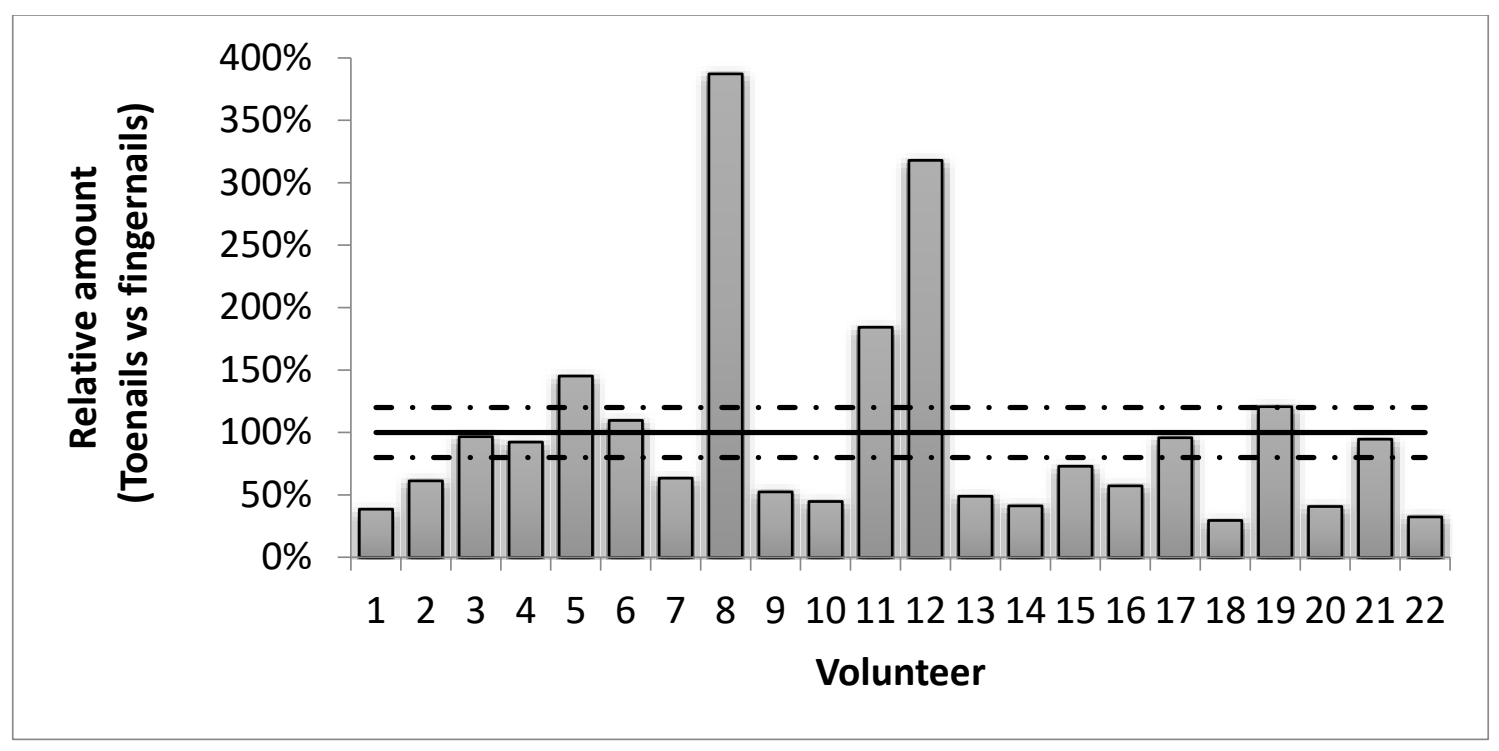

Figure 3. Toenail analysis failed to replace fingernails' melatonin determination. Relative abundance of melatonin levels in toenails compared with the ones of fingernails collected at the same time from several volunteers. Dotted lines represent the $80-120 \%$ threshold required to consider the results as replaceable.

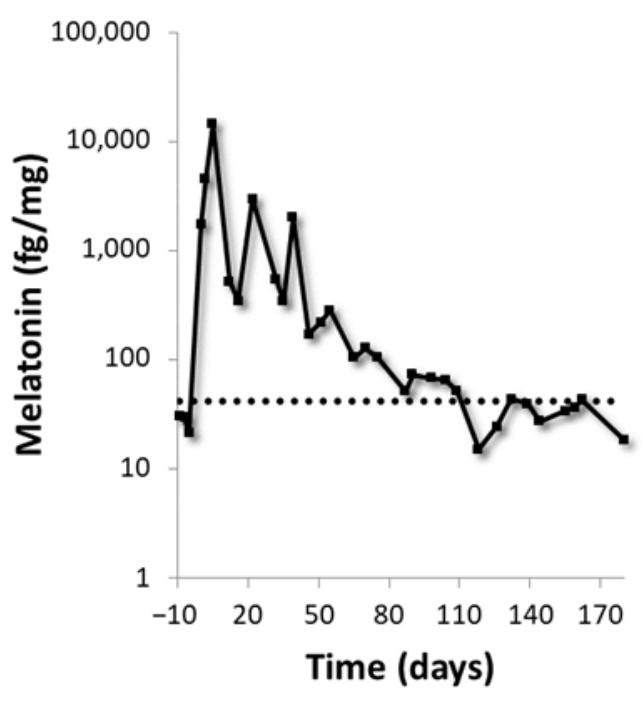

(a)

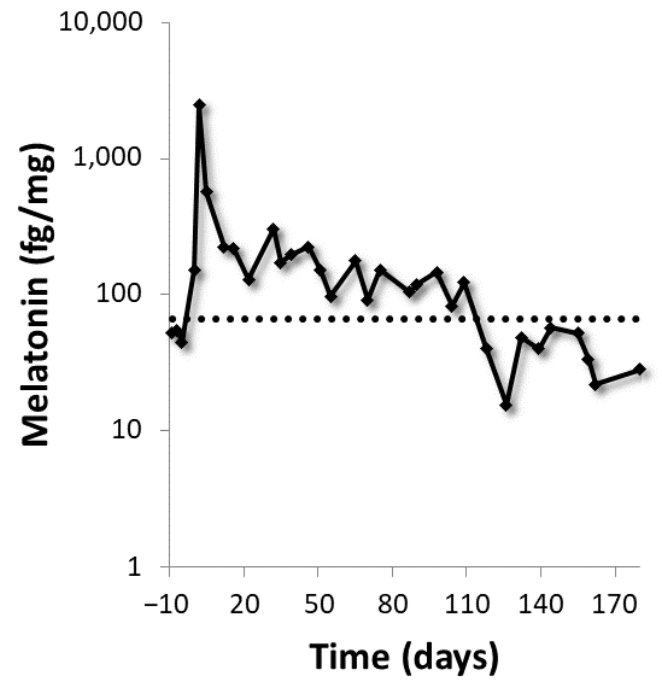

(b)

Figure 4. Nail melatonin content provides a weighted average of the circulating melatonin from the last 3.5 months. Changes over time in the nail's melatonin levels (axis is represented in log scale) due to the exogenous administration of melatonin in the two volunteers: (A) male, (B) female. Dotted lines represent the endogenous levels for each volunteer calculated from basal samples (obtained between days -10 and 0 ).

\section{Discussion}

The potential of nail analysis for determining the global production of melatonin has been evaluated. We have developed and validated a LC-MS/MS analytical method to determine melatonin content in nails. The determination of melatonin in nails is an analytical challenge, since melatonin is present at extremely low concentrations and nails, besides its low sample availability, is a complex matrix.

The current work emphasizes the importance of chromatographic separation in order to separate an interferent ubiquitously present in nails and to provide a reliable quantifi- 
cation of melatonin. It also implies the need for testing the specificity of the method by evaluating the ion ratio between two transitions. This evaluation is difficult when the qualitative transition is clearly less abundant than the quantitative one (like in the case of melatonin). The strategy of adding multiple selected reaction monitoring (SRM) transitions has gained attention over recent years [33,34] for increasing sensitivity. Our results demonstrate that this strategy was useful to improve the sensitivity of the qualitative transition helping in the evaluation of the specificity of the method (Figure S2).

The analytical method was validated by two different approaches. On one hand, melatonin-free matrices were used to establish some parameters, e.g., linearity, accuracy, precision and LOD. However, since melatonin is an endogenous hormone present in each individual, actual blank samples were not available. The use of artificial melatonin-free matrices may underestimate the effect of some matrix components removed during the preparation of melatonin-free nails and for this reason, method validation was complemented by using real matrices to evaluate other parameters such as accuracy, precision and matrix effect. The results of both validation processes indicate that the developed method is suitable for the determination of nail melatonin content.

The evaluation of fingernails' melatonin levels of 84 individuals indicate that nail melatonin content mimics the reported behavior of circulating melatonin. As expected, no significant differences were obtained by gender $[35,36]$ (Figure 2A) but some differences were observed by age (Figure $2 \mathrm{~B}$ ). The variation found in the different age ranges (3-8 fold difference between the maximum and the minimum), specially between 5 to 40 years, are in agreement with previously reports using urinary 6-sulfatoxymelatonin marker for global melatonin production [37] (Figure 2B). Our results agree with the ones obtained by Kennaway et al. which report that melatonin levels decreased mainly in early adulthood [38]. Nail melatonin determination was also able to show the expected decline in melatonin excretion with age $[37,39-42]$. We found a statistically significant decrease (Pearson coefficient $-0.615, p<0.001$ ) of nail melatonin levels with age (Figure $2 \mathrm{C}$ ). The decrease of nail melatonin content observed with age reinforces the interest of using melatonin as aging biomarker $[19,43]$ and opens the possibility of using nail melatonin content as ageing biomarker or even for the evaluation of anti-ageing therapies. In order to confirm that point, a correlation between nail melatonin content and common ageing biomarkers (oxidative stress, RedOx status, mitochondria activity, peroxisome activity, telomere length) [44-46] should be established. Additionally, the confirmation of these results by the analysis of a larger set of samples is advised in order to strengthen the applicability of the nail melatonin content as aging biomarker.

One of the main limitations of using fingernails for analysis is the fact that there is an important number of people with onychophagia (i.e., that bite their fingernails), particularly among the youth population [47]. For these individuals collecting fingernails is not straightforward and the analysis of toenails may be considered as alternative. Unfortunately, we observed that melatonin levels in fingernails and toenails are not comparable (Figure 3, Table S2). Thus, our results seem to advice against the use of toenails as alternative for fingernails. Several potential reasons might be behind these differences. Firstly, toenails grow $30-50 \%$ slower than fingernails [32]. This suggests that the melatonin levels found in both nail types collected at the same time may provide different information. In addition, nails are over-exposed to external factors such as sweat or shampoos. As previously reported for some metabolites in hair [48], this over-exposition might induce some degradation of melatonin in nails. Since the deposition time is longer in toenails than in fingernails, this could partially explain the commonly lower melatonin levels found in toenails. A potential poorer transference/diffusion of melatonin into toenails might also explain the lower levels found compared with fingernails although more experiments would be required to confirm this point.

In order to evaluate the biological meaning of the nail melatonin concentrations, we increased circulating levels of melatonin in two volunteers by the administration of melatonin (daily during 5 consecutive days). The main goal of this pilot study was to 
establish the detection window of this increase by nail analysis. The period in which the increment was detected (from hours to up to three months after the administration) indicates that melatonin is rapidly deposited into nails and quickly diffused through the nail bed. The diffusion of other substance such as methyl red sodium salt or topic drugs in nails has been previously described $[49,50]$. That increase together with the fact that nail melatonin levels gradually decreased to basal levels after 109 days suggested that nails melatonin determination does not capture a fixed moment in time but provides a weighted average of the circulating melatonin in the last 3-4 months.

The developed approach can be also useful to check the adherence of patients/volunteers to treatments based on melatonin administration. Melatonin metabolism in humans is rapid and its half-life varies between 10 and $60 \mathrm{~min}$ after the intake [51]. This fact hinders the evaluation of the adherence of patients/volunteers to melatonin treatments by plasma/saliva analysis. In comparison with these matrices, nail do not require for a fast sample collection after exogenous administration. Our results indicate that nail samples collected at any time will provide robust evidence of exogenous administration.

The proposed strategy is not exempt of limitations. Firstly, we have demonstrated that robust results are only obtained when using fingernails. Therefore, its application to patients/volunteers with onychophagia is rather limited. Additionally, expensive instruments (LC-MS/MS) seem to be required for the determination of the trace levels of nail melatonin. More research is needed in order to evaluate whether more economical approaches are able to determine these levels. Finally, this study did not evaluate the potential effect of some factors such nail dye and collection season in nail melatonin levels

\section{Materials and Methods}

\subsection{Reagents and Chemicals}

Melatonin $\geq 98 \%$ and melatonin-d4 99\% were purchased in Sigma Aldrich (St Louis, MO, USA) and in Toronto Research Chemicals (North York, ON, Canada) respectively. Sodium chloride, potassium carbonate, ethyl acetate and methanol (LC gradient grade) were bought from Merck (Darmstadt, Germany) and ammonium formate (HPLC grade) from Sigma Aldrich (St Louis, MO, USA). Ultrapure water was obtained by a Milli-Q purification system (Millipore Ibérica, Barcelona, Spain).

\subsection{Instrumentation}

LC-MS/MS was used for the analysis (Waters Associates, Milford, MA, USA). The chromatography separation was achieved by an isocratic gradient at $25 \%$ (organic solvent) by using an Acquity CSH C18 column $(100 \mathrm{~mm} \times 2.1 \mathrm{~mm}$ i.d., $1.7 \mu \mathrm{m})$ (Waters Associates, Milford, MA, USA) at a flow rate of $300 \mu \mathrm{L} \mathrm{min}^{-1}$ and water and methanol as mobiles phases (both with ammonium formate $(1 \mathrm{mM})$ and formic acid $(0.01 \% v / v)$ ).

The detection of melatonin was performed in the SRM mode by using the transitions of $233>159$ (collision energy $30 \mathrm{~V}$, monitored 10 times) and $233>174$ (collision energy $10 \mathrm{~V}$ ) for melatonin and $237>163$ (collision energy $30 \mathrm{~V}$ ) and $237>178$ (collision energy $10 \mathrm{~V}$ ) for melatonin-d4. The cone voltage was set at $20 \mathrm{~V}$ and the dwell times at $70 \mathrm{~ms}$.

\subsection{Sample Treatment}

Nail samples were washed with $1 \mathrm{~mL}$ ultrapure water and vortexed for $1 \mathrm{~min}$. The aqueous fraction was discarded, and nail samples were dried overnight. Then, samples were pulverized for 2 min using a ball mill (Mixer Mill MM 200 from Retsch (Haan, Germany)).

After addition of $50 \mu \mathrm{L}$ of melatonin-d4 $\left(500 \mathrm{pg} \mathrm{mL}^{-1}\right), 30 \mathrm{mg}$ of pulverized nails were extracted with $2 \mathrm{~mL}$ of methanol during $1 \mathrm{~h}$ in an ultrasounds bath (Selecta, Barcelona, Spain). Then, samples were centrifuged $(3500 \times g, 5 \mathrm{~min})$ and the methanolic extract was extracted and evaporated under nitrogen stream $\left(40^{\circ} \mathrm{C},<15 \mathrm{psi}\right)$ to dryness. The extract was reconstituted with $100 \mu \mathrm{L}$ of ultrapure water and $10 \mu \mathrm{L}$ were injected into the system. 


\subsection{Preparation of Melatonin-Free Nails}

Melatonin-free matrices were prepared by extracting nail samples twice with methanol as described in Section 4.3. Methanol extracts were discarded and the dry sample was considered as melatonin-free matrix. The absence of melatonin was confirmed by LCMS/MS analysis.

\subsection{Method Validation}

\subsubsection{Method Validation Using Melatonin-Free Nails}

Method validation using melatonin-free nails was performed based on the criteria established on EMA regulations [52]. Briefly, linearity was evaluated by preparing calibration standards in methanol by triplicate at seven different melatonin concentrations (range $6.5-830 \mathrm{fg} / \mathrm{mg}$ ).

Accuracy and precision were evaluated by the analysis of melatonin-free nails spiked at different levels (QC). The method was considered accurate when results were between $80-120 \%$ for the low QC sample and between $85-115 \%$ for medium and high QC samples [52] and precise when CV did not exceed $20 \%$ for low QC and $15 \%$ for medium and high QC [52].

The LOD was defined as the estimated providing a signal to noise ratio $(\mathrm{S} / \mathrm{N})$ of 3 . The lowest limit of quantification (LLOQ) and the upper limit of quantification (ULOQ) were the lowest and the highest value described to be accurate $(80-120 \%$ and $85-115 \%$ respectively) and precise (CVs $<20 \%$ and $<15 \%$ respectively).

\subsubsection{Method Validation Using Real Nail Matrix}

Validation using real nail matrix was performed by standard addition as previously reported by our group $[53,54]$.

Method accuracy and precision were also evaluated by performing standard addition in real nail matrices $(n=6)$ The method was considered accurate and precise if intra-assay and inter-assay accuracy ranged $80-120 \%$ and CVs were below $20 \%$.

Matrix effect of real nails was calculated in terms of recovery by comparing the slopes obtained in the standard addition and the solvent standards. The recovery was calculated as "Recovery $=$ (slope standard addition $) /$ (slope in solvent $) \times 100$ " and was considered satisfactory in the range of $80-120 \%$ and CV below $20 \%$.

Specificity was tested in real nails $(n=5)$ by comparing the ion ratio between the two selected transitions obtained in human samples with the one obtained in methanolic solutions $(n=7)$. Differences below $20 \%$ in ion ratio comparison were considered as indicative of the absence of significant interferences in real nail samples.

\subsection{Method Application}

The applicability of the method was evaluated by 3 different experiments. Firstly, 84 fingernails' samples selected from males and females and at different range ages (described in Section 4.7.2) were evaluated to establish the endogenous melatonin levels in fingernails. Secondly, 22 volunteers that provide fingernails also provided toenails' samples and a comparison between both nail samples was performed to evaluate the possibility to use toenails instead of fingernails. Then, two volunteers were administered by melatonin during 5 days and the effect of melatonin administration in melatonin fingernails levels was studied.

\subsection{Samples}

The free margin of nail samples was collected by scissor cutting and stored at room temperature until analysis. The study was performed according to the principles outlined by the Helsinki Declaration and informed consent was obtained from all volunteers. 


\subsubsection{Method Validation}

Nail samples from 6 healthy volunteers (ages from 5 to 60, not taking exogenous melatonin) were collected for 2 months and combined (one sample per volunteer).

\subsubsection{Method Application \\ Endogenous Melatonin Levels in Fingernails}

For establishment of endogenous levels, fingernail samples were collected from 84 healthy volunteers (50 females (ages 5-96 years old), 34 males (ages 5-79 years old)).

Comparison of Melatonin Levels between Fingernails and Toenails

Twenty-two of the aforementioned volunteers also provided toenail samples (13 females and 9 males, ages $5-75$ years old).

\section{Effect of Melatonin Administration in Melatonin Fingernails' Levels}

In order to have a first estimation of the detection time window, a pilot study was performed. Two healthy volunteers (one female, 44 years old; one male, 44 years old) orally took one melatonin tablet (1.9 mg tablets from ESI melatonin Pure, ESI srl, Italy) daily during 5 consecutive days (administration time around $2 \mathrm{~h}$ before going bed). Nail samples from 3-4 fingers were collected as described in Figure S2. Briefly, 3 samples were collected before administration to establish basal levels and subsequently one sample was collected every 2-18 days for 180 days starting the day after the first administration.

\subsection{Data Analysis}

Quantification of melatonin was performed using MassLynx software (version 4.1) from Waters Associates, Milford, MA, USA.

The statistical analysis was performed by the SPSS software (version 18.0; IBM, Armonk, New York, NY, USA). The influence of gender and age in the fingernail's melatonin was evaluated by a two-way ANOVA and the influence of age in the fingernail's melatonin was determined by a one-way ANOVA with a Bonferroni post-hoc test (significant levels was considered when $p<0.05$ ). The Pearson's correlation with a two-tailed test was performed to assess the correlations between fingernails' melatonin and age (significant level at $p<0.05)$.

In order to estimate the effect of melatonin administration, the detection window was estimated by evaluating the period in which the melatonin levels exceeded the basal ones. Briefly, an individual threshold value for melatonin was calculated for each volunteer as Threshold $=$ (mean melatonin in basal samples) $+3 \times$ (standard deviation in basal samples). The detection window for each volunteer was defined as the period in which values exceeded the individual threshold $[55,56]$.

\section{Conclusions}

The potential of nail analysis for determining the global production of melatonin has been evaluated. Our results indicate that fingernail melatonin content mimics the expected behavior of circulating melatonin. Therefore, the determination of nail melatonin content may be a promising tool for obtaining information about the global production of melatonin and pineal gland status. This strategy might reveal new insights into some pathological states with disturbances in melatonin production.

Supplementary Materials: The following are available online at https: / /www.mdpi.com/1422-0 067/22/2/921/s1, Figure S1: Chromatogram representation of SRM transitions of melatonin in a real nail sample (18 fg/mg). (A) Melatonin-d4, (B) Melatonin (273 > 174 transition, quantitative), (C) Melatonin (summation of $273>159$ transition, qualitative) and (D) Melatonin (single $273>159$ transition, qualitative), Figure S2: General overview of the design and schedule of the study of melatonin administration. (A) Distribution of the different collected (a) nails collected from both little fingers and both ring fingers (4 nails), (b) nails collected from right thumb and left index finger and 
middle finger (3 nails) and (c) nails collected from left thumb and right index finger and middle finger (3 nails); (B) Schedule of samples collection (in orange sample collection (a), in blue sample collection (b) and in red sample collection (c)). In dots, days that exogenous melatonin was administered to volunteers are highlighted, Table S1: Melatonin levels (in $\mathrm{fg} / \mathrm{mg}$ ) in the selected population. Abbreviations: F: female and M: male, Table S2: Melatonin levels (in fg/mg) in fingernails and toenails. Abbreviations: F: female and M: male.

Author Contributions: A.G.-G., conceptualization, methodology, investigation, validation, formal analysis, visualization and writing-original draft; B.M.-S.-M., methodology, investigation and validation; N.H., methodology and investigation; O.J.P., conceptualization, methodology, investigation, validation, formal analysis, visualization, writing —original draft, supervision and funding acquisition. All authors have read and agreed to the published version of the manuscript.

Funding: This work was supported by grants from Instituto de Salud Carlos III-FEDER (PI18/00048).

Institutional Review Board Statement: The study was conducted according to the guidelines of the Declaration of Helsinki, and approved by the Comitè Ètic d'Investigació Clínica del Parc de Salut Mar (protocol code 2018/7952/I, approval date 12 February 2019).

Informed Consent Statement: Informed consent was obtained from all subjects involved in the study.

Data Availability Statement: The data presented in this study are available on request from the corresponding author.

Acknowledgments: Our sincere thanks to the volunteers who decided to participate in the study.

Conflicts of Interest: The authors declare no conflict of interest.

\begin{tabular}{ll}
\multicolumn{2}{l}{ Abbreviations } \\
DLMO & $\begin{array}{l}\text { Dim light melatonin onset } \\
\text { Liquid chromatography tandem mass spectrometry } \\
\text { LC-MS/MS } \\
\text { LOD }\end{array}$ \\
LV & Coefficient of variation \\
CDER & Center for Drug Evaluation and Research \\
SRM & Selected reaction monitoring \\
QC & Melatonin-free nails spiked at different levels \\
S/N & Signal to noise ratio
\end{tabular}

\section{References}

1. Kennaway, D.J. Measuring melatonin by immunoassay. J. Pineal Res. 2020, 69, e12657. [CrossRef]

2. Reiter, R.J.; Tan, D.-X.; Mayo, J.C.; Sainz, R.M.; Leon, J.; Czarnocki, Z. Melatonin as an antioxidant: Biochemical mechanisms and pathophysiological implications in humans. Acta Biochim. Pol. 2003, 50, 1129-1146. [CrossRef]

3. Tan, D.-X.; Chen, L.D.; Poeggeler, B.; Manchester, L.C. Melatonin: A potent endogenous hydroxyl radical scavenger. Endocr. J. 1993, 1, 57-60.

4. Galano, A.; Tan, D.X.; Reiter, R.J. On the free radical scavenging activities of melatonin's metabolites, AFMK and AMK. J. Pineal Res. 2013, 54, 245-257. [CrossRef]

5. Barrenetxe, J.; Delagrange, P.; Martínez, J.A. Physiological and metabolic functions of melatonin. J. Physiol. Biochem. 2004, 60, 61-72. [CrossRef]

6. Carrillo-Vico, A.; Lardone, P.J.; Álvarez-Sánchez, N.; Rodríguez-Rodríguez, A.; Guerrero, J.M. Melatonin: Buffering the Immune System. Int. J. Mol. Sci. 2013, 14, 8638-8683. [CrossRef] [PubMed]

7. Olcese, J. Melatonin and Female Reproduction: An Expanding Universe. Front. Endocrinol. 2020, 11, 85. [CrossRef] [PubMed]

8. Carlomagno, G.; Minini, M.; Tilotta, M. Vittorio Vittorio Unfer for The Experts Group on Inositol in Basic and Clinical Research From Implantation to Birth: Insight into Molecular Melatonin Functions. Int. J. Mol. Sci. 2018, 19, 2802. [CrossRef] [PubMed]

9. Skene, D.; Vivien-Roels, B.; Sparks, D.; Hunsaker, J.; Pévet, P.; Ravid, D.; Swaab, D. Daily variation in the concentration of melatonin and 5-methoxytryptophol in the human pineal gland: Effect of age and Alzheimer's disease. Brain Res. 1990, 528, 170-174. [CrossRef]

10. Uchida, K.; Okamoto, N.; Ohara, K.; Morita, Y. Daily rhythm of serum melatonin in patients with dementia of the degenerate type. Brain Res. 1996, 717, 154-159. [CrossRef]

11. Aoki, M.; Yokota, Y.; Hayashi, T.; Kuze, B.; Murai, M.; Mizuta, K.; Ito, Y. Disorder of the saliva melatonin circadian rhythm in patients with Meniere's disease. Acta Neurol. Scand. 2006, 113, 256-261. [CrossRef] [PubMed] 
12. Hu, S.; Shen, G.; Yin, S.; Xu, W.; Hu, B. Melatonin and tryptophan circadian profiles in patients with advanced non-small cell lung cancer. Adv. Ther. 2009, 26, 886-892. [CrossRef] [PubMed]

13. Grin, W.; Grünberger, W. A significant correlation between melatonin deficiency and endometrial cancer. Gynecol. Obstet. Investig. 1998, 45, 62-65. [CrossRef] [PubMed]

14. Peschke, E.; Stumpf, I.; Bazwinsky, I.; Litvak, L.; Dralle, H.; Mühlbauer, E. Melatonin and type 2 diabetes ? A possible link? J. Pineal Res. 2007, 42, 350-358. [CrossRef]

15. Hardeland, R. Neurobiology, Pathophysiology, and Treatment of Melatonin Deficiency and Dysfunction. Sci. World J. 2012, 2012, 1-18. [CrossRef]

16. Puy, H.; Deybach, J.-C.; Baudry, P.; Callebert, J.; Touitou, Y.; Nordmann, Y. Decreased nocturnal plasma melatonin levels in patients with recurrent acute intermittent porphyria attacks. Life Sci. 1993, 53, 621-627. [CrossRef]

17. Acuña-Castroviejo, D.; Escames, G.; Reiter, R.J. Melatonin therapy in fibromyalgia. J. Pineal Res. 2005, 40, 98-99. [CrossRef]

18. Monteleone, P.; Martiadis, V.; Maj, M. Circadian rhythms and treatment implications in depression. Prog. Neuro Psychopharmacol. Biol. Psychiatry 2011, 35, 1569-1574. [CrossRef]

19. Karasek, M. Melatonin, human aging, and age-related diseases. Exp. Gerontol. 2004, 39, 1723-1729. [CrossRef]

20. Armstrong, S.; Redman, J. Melatonin: A chronobiotic with anti-aging properties? Med. Hypotheses 1991, 34, 300-309. [CrossRef]

21. Karasek, M.; Reiter, R.J. Melatonin and aging. Neuro Endocrinol. Lett. 2002, 23, 14-16.

22. Luo, F.; Sandhu, A.F.; Rungratanawanich, W.; Williams, G.E.; Akbar, M.; Zhou, S.; Song, B.; Wang, X. Melatonin and Autophagy in Aging-Related Neurodegenerative Diseases. Int. J. Mol. Sci. 2020, 21, 7174. [CrossRef] [PubMed]

23. Jenwitheesuk, A.; Nopparat, C.; Mukda, S.; Wongchitrat, P.; Govitrapong, P. Melatonin Regulates Aging and Neurodegeneration through Energy Metabolism, Epigenetics, Autophagy and Circadian Rhythm Pathways. Int. J. Mol. Sci. 2014, 15, 16848-16884. [CrossRef]

24. De Almeida, E.A.; Di Mascio, P.; Harumi, T.; Spence, D.W.; Moscovitch, A.; Hardeland, R.; Cardinali, D.P.; Brown, G.M.; Pandi-Perumal, S.R. Measurement of melatonin in body fluids: Standards, protocols and procedures. Child's Nerv. Syst. 2011, 27, 879-891. [CrossRef]

25. Kennaway, D.J. A critical review of melatonin assays: Past and present. J. Pineal Res. 2019, 67, e12572. [CrossRef] [PubMed]

26. Lockley, S.W. Journal of Pineal Research guideline for authors: Measuring melatonin in humans. J. Pineal Res. 2020, 69, e12664. [CrossRef] [PubMed]

27. Graham, C.; Cook, M.R.; Kavet, R.; Sastre, A.; Smith, D.K. Prediction of nocturnal plasma melatonin from morning urinary measures. J. Pineal Res. 1998, 24, 230-238. [CrossRef] [PubMed]

28. Saksvik-Lehouillier, I.; Harrison, S.L.; Marshall, L.M.; Tranah, G.J.; Ensrud, K.; Ancoli-Israel, S.; Clemons, A.; Redline, S.; Stone, K.L.; Schernhammer, E.S.; et al. Association of Urinary 6-Sulfatoxymelatonin (aMT6s) Levels and Objective and Subjective Sleep Measures in Older Men: The MrOS Sleep Study. J. Gerontol. Ser. A Boil. Sci. Med Sci. 2015, 70, 1569-1577. [CrossRef]

29. Stebelova, K.; Roska, J.; Zeman, M. Impact of Dim Light at Night on Urinary 6-Sulphatoxymelatonin Concentrations and Sleep in Healthy Humans. Int. J. Mol. Sci. 2020, 21, 7736. [CrossRef]

30. Palmeri, A.; Pichini, S.; Pacifici, R.; Zuccaro, P.; Lopez, A. Drugs in Nails. Clin. Pharmacokinet. 2000, 38, 95-110. [CrossRef]

31. Wu, H.; Zhou, K.; Xu, P.; Xue, J.; Xu, X.; Liu, L. Associations of perceived stress with the present and subsequent cortisol levels in fingernails among medical students: A prospective pilot study. Psychol. Res. Behav. Manag. 2018, 11, 439-445. [CrossRef] [PubMed]

32. Shu, I.; Negrusz, A.; Jones, J.; Lewis, U. Detection of Drugs in Nails: Three Year Experience. J. Anal. Toxicol. 2015, 39, 624-628. [CrossRef] [PubMed]

33. Vitku, J.; Skodova, T.; Sosvorova, L.; Hampl, R.; Hill, M.; Heracek, J.; Bicikova, M.; Stárka, L. Development and validation of LC-MS/MS method for quantification of bisphenol A and estrogens in human plasma and seminal fluid. Talanta 2015, $140,62-67$. [CrossRef] [PubMed]

34. Li, X.; Li, S.; Kellermann, G. Simultaneous determination of three estrogens in human saliva without derivatization or liquidliquid extraction for routine testing via miniaturized solid phase extraction with LC-MS/MS detection. Talanta 2018, 178, 464-472. [CrossRef] [PubMed]

35. Nathan, P.J.; Wyndham, E.L.; Burrows, G.D.; Norman, T.R. The effect of gender on the melatonin suppression by light: A dose response relationship. J. Neural Transm. 2000, 107, 271-279. [CrossRef] [PubMed]

36. Boivin, D.B.; Shechter, A.; Boudreau, P.; Begum, E.A.; Ying-Kin, N.M.K.N. Diurnal and circadian variation of sleep and alertness in men vs. naturally cycling women. Proc. Natl. Acad. Sci. USA 2016, 113, 10980-10985. [CrossRef]

37. Mahlberg, R.; Tilmann, A.; Salewski, L.; Kunz, D. Normative data on the daily profile of urinary 6-sulfatoxymelatonin in healthy subjects between the ages of 20 and 84. Psychoneuroendocrinology 2006, 31, 634-641. [CrossRef]

38. Kennaway, D.J.; Lushington, K.; Dawson, D.; Lack, L.; Heuvel, C.V.D.; Rogers, N. Urinary 6-sulfatoxymelatonin excretion and aging: New results and a critical review of the literature. J. Pineal Res. 1999, 27, 210-220. [CrossRef]

39. Sack, R.L.; Lewy, A.J.; Erb, D.L.; Vollmer, W.M.; Singer, C.M. Human Melatonin Production Decreases With Age. J. Pineal Res. 1986, 3, 379-388. [CrossRef]

40. Wetterberg, L.; Bergiannaki, J.D.; Paparrigopoulos, T.; Von Knorring, L.; Eberhard, G.; Bratlid, T.; Yuwiler, A. Normative melatonin excretion: A multinational study. Psychoneuroendocrinology 1999, 24, 209-226. [CrossRef]

41. Skene, D. Melatonin rhythmicity: Effect of age and Alzheimer's disease. Exp. Gerontol. 2003, 38, 199-206. [CrossRef] 
42. Wu, Y.-H.; Swaab, D.F. The human pineal gland and melatonin in aging and Alzheimer's disease. J. Pineal Res. 2005, 38, 145-152. [CrossRef] [PubMed]

43. Bubenik, G.A.; Konturek, S.J. Melatonin and aging: Prospects for human treatment. J. Physiol. Pharmacol. 2011, 62, 13-19. [PubMed]

44. Venkataraman, K.; Khurana, S.; Tai, T.C. Oxidative Stress in Aging-Matters of the Heart and Mind. Int. J. Mol. Sci. 2013, 14, 17897-17925. [CrossRef]

45. Shekhidem, H.A.; Sharvit, L.; Leman, E.; Manov, I.; Roichman, A.; Holtze, S.; Huffman, D.M.; Cohen, H.Y.; Hildebrandt, T.; Shams, I.; et al. Telomeres and Longevity: A Cause or an Effect? Int. J. Mol. Sci. 2019, 20, 3233. [CrossRef]

46. Webb, M.; Sideris, D.P. Intimate Relations-Mitochondria and Ageing. Int. J. Mol. Sci. 2020, 21, 7580. [CrossRef]

47. Ellington, E. Chronic Nail Biting in Youth. J. Psychosoc. Nurs. Ment. Health Serv. 2017, 55, 23-26. [CrossRef]

48. Dong, Z.; Wang, C.; Zhang, J.; Wang, Z. A UHPLC-MS/MS method for profiling multifunctional steroids in human hair. Anal. Bioanal. Chem. 2017, 409, 4751-4769. [CrossRef]

49. Palliyil, B.B.; Li, C.; Owaisat, S.; Lebo, D.B. Lateral Drug Diffusion in Human Nails. Ageing Int. 2014, 15, 1429-1438. [CrossRef]

50. Saner, M.V.; Kulkarni, A.D.; Pardeshi, C.V. Insights into drug delivery across the nail plate barrier. J. Drug Target. 2014, 22, 769-789. [CrossRef]

51. Peuhkuri, K.; Sihvola, N.; Korpela, R. Dietary factors and fluctuating levels of melatonin. Food Nutr. Res. 2012, 56, 17252. [CrossRef] [PubMed]

52. European Medicines Agency. Guideline on bioanalytical method validation. EMA Guidel. 2012, 44, 1-23.

53. Olesti, E.; Rodríguez-Morató, J.; Gomez-Gomez, A.; Ramaekers, J.G.; de la Torre, R.; Pozo, O.J. Quantification of endogenous neurotransmitters and related compounds by liquid chromatography coupled to tandem mass spectrometry. Talanta 2019, 192, 93-102. [CrossRef] [PubMed]

54. Gomez-Gomez, A.; Miranda, J.; Feixas, G.; Betegon, A.A.; Crispi, F.; Gratacós, E.; Pozo, O.J. Determination of the steroid profile in alternative matrices by liquid chromatography tandem mass spectrometry. J. Steroid Biochem. Mol. Biol. 2020, 197, 105520. [CrossRef] [PubMed]

55. Kotronoulas, A.; Gomez-Gomez, A.; Fabregat, A.; Segura, J.; Yang, S.; Xing, Y.; Moutian, W.; Marcos, J.; Joglar, J.; Ventura, R.; et al. Evaluation of markers out of the steroid profile for the screening of testosterone misuse. Part I: Transdermal administration. Drug Test. Anal. 2017, 10, 821-831. [CrossRef]

56. Kotronoulas, A.; Gomez-Gomez, A.; Fabregat, A.; Segura, J.; Yang, S.; Xing, Y.; Moutian, W.; Marcos, J.; Joglar, J.; Ventura, R.; et al. Evaluation of markers out of the steroid profile for the screening of testosterone misuse. Part II: Intramuscular administration. Drug Test. Anal. 2017, 10, 849-859. [CrossRef] 